\title{
HISTORIA INSTANTÁNEA. EL NUEVO IMPULSO HISTORIOGRÁFICO FRANCÉS O HISTORIA MUNDIAL EN LOS TIEMPOS DE TWITTER
}

INSTANT HISTORY. THE NEW FRENCH HISTORIOGRAPHY
AND WORLD HISTORY IN THE TIMES OF TWITTER

\author{
Daniele Conversi \\ Ikerbasque, UPV/EHU
}

ORCID: https://orcid.org/0000-0002-6618-2738

Recibido el 16-5-2018 y aceptado el 14-6-2018

Resumen: Una nueva generación de historiadores encabezada por Patrick Boucheron ha desafiado la noción de una identidad francesa fija y primordial. En su lugar, una nueva historia «interconectada» persigue su vocación global anclándose al mismo tiempo en lo nacional.

La secuencia cronológica de Boucheron y colegas desentierra una red de narrativas nacionales, co-nacionales y transnacionales canalizadas según fechas escogidas en base a la creatividad y la curiosidad, como destellos en el firmamento del tiempo para orientarse en la noche oscura de las historiografías «nacionalizadas».

Con su ritmo cronológico marcado por eventos altamente simbólicos , la innovación no reside tanto en un nuevo giro metodológico, cuanto en un cambio de paradigma presentacional. Lo que hace la novedad es el hilo de diferentes tramas e historias. Así, la historia de Francia se convierte en una encrucijada de encuentros, una confluencia de casualidades, señalando al mismo tiempo los límites del deconstrucciónismo /constructivismo en la historia contemporánea: Ya no es suficiente deconstruir la narrativa patriótica si las deconstrucciones pueden ser apropiadas para deconstruir arbitrariamente hechos reales como si fueran «noticias falsas». Y si la deconstrucción se puede derivar en la construcción arbitraria, el deconstructivismo se deconstruye a sí mismo. Superando el constructivismo-deconstruccionismo se intenta así producir una nueva «historia instantánea». De esta forma, podemos gozar de nuevas maneras de re-concebir la reconfiguración de la misma identidad nacional en una dimensión mas internacional, con la elección de instantes o momentos de cambios simbólicos, culturales y políticos que imparten su huella a través de las generaciones.

Palabras clave: Nueva historiografía, historia de Francia, nacionalismo, identidad nacional. 


\begin{abstract}
A new generation of historians led by Patrick Boucheron has challenged the notion of a fixed and primordial French identity. Instead, it is claimed that a new «interconnected» story can pursue its global vocation by simultaneously anchoring itself in the national. The chronological sequence of Boucheron and colleagues unearths a network of national, co-national and transnational narratives channelled according to dates chosen on the basis of creativity and curiosity, as flashes in the firmament of time to orient the reader in the dark night of «nationalized» historiographies.

With its chronological rhythm marked by highly symbolic events, innovation lies not so much in a new methodological turn, but in a change of presentational paradigm. The novelty lies in the juxtaposition of different plots and stories, so that the history of France becomes a crossroads of encounters and a confluence of coincidences. At the same time, the work points out the limits of deconstructionism-constructivism in contemporary history: if deconstruction can be appropriated to arbitrarily deconstruct objective facts to make them «fake news», it is no longer sufficient to deconstruct the patriotic narrative. And, once deconstruction results in arbitrary construction, deconstructivism deconstructs itself. The effort to overcome the constructivism-deconstructionism framework has thus produced a new «instant history». In this way, we can appreciate new paths of reconceiving and reconfiguring national identity in a more international fashion, with the condensed choice of «instants» as moments of symbolic, cultural and political change that make their mark through the generations.
\end{abstract}

Keywords: New Historiography, History of France, nationalism, national identity, World History, new French historiography, global history. 
Boucheron, Patrick (ed.), Histoire mondiale de la France [Historia Global de Francia], Paris: Éditions du Seuil, 2017, 797 pp.

La noción de una identidad francesa fija y primordial ha sido desafiada por una nueva generación de historiadores franceses: La Histoire mondiale de la France editada por Patrick Boucheron (2017) reúne a 122 colaboradores que se centran en 146 fechas clave de eventos históricos en otros tantos capítulos. Cada uno ha sido confiado a un experto, a menudo relativamente joven (excepto algunos profesores eméritos). Cada capítulo, adornado sencillamente con una condensada bibliografía y sin notas, combina en 4 a 5 páginas un enfoque cronológico con una perspectiva contemporánea 'transnacional'. Por cierto, 'transnacional' no significa imperial o un anhelo por la misión civilizadora de la France universelle et éternelle, sino, todo lo contrario, una historia 'cruzada' o historia 'interconectada', o sea una historia vocacionalmente global, aunque firmemente anclada en lo nacional.

Como cada capítulo está dedicado a una fecha específica, la primera pregunta que surge es: ¿Cómo se han elegido estas fechas? La respuesta parece ser: para inspirar, incluso provocar o sacudir el imaginario colectivo, evocando un nuevo conjunto de eventos fundacionales.

Por ejemplo, ¿cuán importante fue la adquisición del Atlas Catalán (L'Atles català) por el rey Carlos V de Francia en 1380 para la Biblioteca Real (ahora Bibliothèque nationale de France, BnF), donde todavía está? Suficientemente como para merecer una entrada propia, donde explica que se trataba de un enorme mapa iluminado del «mundo conocido», probablemente dibujado en 1375 por el cartógrafo judío Cresques Abraham de la Escuela Cartográfica de Mallorca, en la practica la síntesis de un mappae mundi medieval centrado en Jerusalén.

Casi todas las fechas elegidas en la cronología son intrigantemente inspiradoras, revelando nuevos escenarios transculturales en una secuencia formidable de 40,000 años de historia «francesa», enriquecida por anécdotas ingeniosas, donde la simplicidad de lo ordinario supera la trivialidad de lo extraordinario.

Esta es una salida incisiva de la narrativa contigua y fluida propia de la longue durée prevista por la Escuela de los Annales (École des Annales), lanzada a fines de la década de 1920 por Lucien Febvre y Marc Bloch. Aquí no encontramos una narración de longue durée, pero sí una larga historia de Francia. Significativamente, todo empieza con la Revolución Neolítica, descrita como la ruptura más grande en la historia mun- 
dial, con el surgimiento emergente de la agricultura, la domesticación de los animales y la sedentarización, pero llegando a Europa solo 3.000 años después de su primera aparición en el Medio Oriente. El advenimiento de la modernidad industrial del siglo XVIII no se considera tan indiscutiblemente fundamental como el advenimiento del neolítico.

El volumen rechaza así elegantemente el cliché de «nuestros ancestros los galos» y, en general, la noción de una identidad francesa que existe desde tiempos inmemoriales. En su lugar aparece el «Hombre de CroMagnon», el primer Homo sapiens sapiens del Paleolítico superior, cuyo esqueleto fosilizado fue descubierto en 1868 en Les Eyzies (Dordogne) y con fecha de carbono 14 de aproximadamente 28.000 años antes. En lugar de soberanos francos y tribus galas, nuestros comunes ancestros nómadas cazadores-recolectores nos encantan con sus intrincados rituales de caza, nacimiento y muerte, con su mezcla de materiales raciales y genéticos de diversas procedencias, una tendencia hacia el mestizaje que persistió durante los siguientes 36.000 años. Luego, los estilos de vida universales de los cazadores-recolectores reinaban en todo el planeta en un equilibrio óptimo con el entorno natural circundante. Por lo tanto, Francia no fue «francesa» desde el fondo de la historia, por lo que el antiguo mito milenario de los orígenes pierde su encanto. Aquí me limitaré a evaluar algunas de las paginas del libro que tratan mas bien de eventos relacionados con la historia contemporánea y presente.

En Capital in the Twenty-First Century, el historiador económico Thomas Piketty traza paralelos inquietantes entre el Belle Époque, que precedió a la Primera Guerra Mundial, y nuestra edad de consumo masivo y sobreexplotación acompañada de una brecha cada vez mayor entre ricos y pobres (Piketty 2014). Sin embargo, encontramos este tema crucial solo brevemente en un capítulo (sobre el año 1892) dedicado a la explosión del terrorismo con la estrategia anarquista de la «propaganda por el hecho», o «por el acto» (propagande par le fait), donde se menciona solo brevemente el «círculo vicioso de ataque-represión-ataque /reacción» (540), conectándolo apenas con la creciente brecha de desigualdad en un momento de rápidas revoluciones tecnológicas y de expansión del poder centralizado.

Otra entrada explora la insolvencia financiera de la Exposition universelle de París (1900) con más de 48 millones de visitantes de todo el mundo, pero incapaz de generar un balance presupuestario en positivo. La Expo destacó los avances tecnológicos asombrosamente rápidos de Francia junto con su supremacía militar, sin casi eclipsar a los de las potencias 
imperiales rivales. Al igual que muchas otras Expos, la de 1900 también acentuó las visiones esencialistas de la nación, en la que cada pabellón representaba una simplificación de las tradiciones culturales y de la ciencia «nacional», asumiendo la homogeneidad interna de cada «estado-nación» allí representado, encarnando plenamente los procesos de homogenización cultural característicos tanto del «largo siglo XIX» como del «corto siglo XX» (Archilés Cardona and García Carrión 2012; Conversi 2012).

Después de unas cuantas citas más, de repente nos vemos arrojados a la caldera de 1914, sin apenas explicación previa del pedigrí cultural y tecno-político que nos lleva a la Primera Guerra Mundial. El capítulo es lo suficientemente equilibrado como para no centrarse en el martirio francés, sino más bien en las gigantescas bajas sufridas por los Aliados en Francia, en particular las tropas inglesas. También examina la variada procedencia étnica y regional de las tropas, regimientos y combatientes, revelando una faceta «cosmopolita» apenas conocida de la Primera Guerra Mundial, hasta la ofensiva del Somme (1 de julio a 18 de noviembre de 1916).

El siguiente capítulo está consagrado a la rebelión anticolonial de 1917 de los Kanaks (Melanesios indígenas) en la Nueva Caledonia francesa. Más mundanamente, 1921 se dedica, en cambio, al lanzamiento de la fragancia Chanel N. 5 por la diseñadora de alta costura Gabrielle «Coco» Chanel (1883-1971).

Varias fechas funcionan como señales. De gran interés, el año 1923 está consagrado a un evento desconocido, pero altamente significativo: la llegada a Francia de la familia Aznavourian escapando del genocidio armenio desatado por los Jóvenes Turcos a partir de 1915 (Akçam 2012; 2013; Schaller and Zimmerer 2009; Üngör 2011; Üngör and Polatel 2011; Weitz 2011). Su hijo, Shahnour Vaghinag Aznavourian, nacido el 22 de mayo de 1924, se hizo famoso mundialmente como cantante y compositor con el nombre de Charles Aznavour, uno de los destacados protagonistas de la chanson française. La idea de Francia como terre d'accueil (tierra de acogida) y terre d'asile (tierra de asilo) se explora nuevamente a través de un cautivador capítulo sobre «el otro 11 de septiembre», el de 1973, cuando el gobierno de Salvador Allende (1908-1973) en Chile fue derrocado por un golpe militar encabezado por Estados Unidos, mientras que Francia dio la bienvenida a 10.000 refugiados chilenos.

Algunas fechas están impregnadas de profunda resonancia simbólica y emocional. La muerte inesperada de Michael Foucault en 1984, a los 53 años de edad, tiene pocos paralelismos en la historia traumática intelectual, quizás una herida sólo superada por el asesinato, todavía envuelto 
en el misterio, del poeta, intelectual y director de cine italiano Pier Paolo Pasolini en 1975 en el litoral romano. Foucault se explora aquí a la luz de sus últimos escritos, a veces póstumos, sobre todo su abierta empatía con la primera fase de la Revolución Shi'a de 1979 en Irán, de una manera que la entrada funciona como un puente para comprender el Medio Oriente, en contraste con el habitual cierre nacionalista contra «el resto».

La secuencia cronológica desentierra una red de narrativas nacionales, co-nacionales y transnacionales canalizadas por la creatividad, la curiosidad y una amplia visión, como destellos en el firmamento del tiempo que ayudan a irradiar la oscura noche de las historiografías «nacionalizadas».

Con su emblemático simbolismo cronológico, la novedad en realidad no reside en un nuevo giro metodológico. De alguna manera, el método de Boucheron puede describirse más bien como «anti-método», por entonces más cercano a la anarquía epistemológica de Paul Feyerabend, con sus reservas sobre la posibilidad contemporánea de un método científico propio de la revolución científica que inauguró la era temprano-moderna (Feyerabend 1975). En lugar de una nueva metodología, tenemos aquí un cambio de presentación, sin la pretensión de descubrir un lienzo más amplio dentro de una narrativa fluida y continuada. Lo que hace la novedad es, en cambio, el hilo de diferentes tramas e historias, sin sucumbir a la tentación de moldear un crisol de narrativas idiosincrásicas.

Por lo tanto, la historia de Francia se convierte en una encrucijada de encuentros, una confluencia de casualidades - en lugar de la unidad monótona y ficticia del Hexágono concebida por los historiadores oficiales a-la Pierre Nora - y aún discernible en los campos de la 'historia cultural', la 'historia de las representaciones', o el 'histoire des mentalités'. De hecho, la llamada nouvelle histoire de la tercera generación de los Annales comenzó en la década de 1970 con Jacques Le Goff y es, quizás incorrectamente, identificada como el 'giro cultural' de la historia. Sin embargo, este último se ha revelado como una sociedad cerrada, caracterizada en forma privada por un conjunto de relaciones de parentesco e incluso matrimonios entre sus historiadores más prominentes, que a su vez se cruzan con destacados políticos (Burke 2015: ch. 4). Por lo tanto, la novedad ofrecida por Boucheron y sus colegas es más que bienvenida en varias áreas y por múltiples razones.

Esa nouvelle vague también señala los límites de la deconstrucción en la historia contemporánea, justo cuando ésta ha alcanzado sus límites externos con demasiada facilidad y de forma normalizada. Tomando prestadas las palabras de Mikhail Bakhtin (1895-1975), el deconstructivismo se ha con- 
vertido en un «discurso de doble voz»: Típicamente, las nociones deconstructivistas militantes como la «invención de la tradición» de Hobsbawm han sido apropiadas e incorporadas a los discursos nacionalistas (Hobsbawm and Ranger 2013). Así, en Italia, la noción de «invención de la Padania» de la Liga Norte ha sido utilizada explícita y orgullosamente por sus militantes (Oneto 1997). El proceso se ha profundizado en una era vilipendiada por la omnipresencia de «post-verdad» y «noticias falsas» en línea, así como la negación del cambio climático está cada vez más relacionada con la «deconstrucción» del análisis científico. En otras palabras, la mayoría de las deconstrucciones, aunque pedantes, tediosas e informadas por la crítica literaria o la análisis del discurso, pueden ser apropiadas para deconstruir arbitrariamente hechos reales como si fueran noticias falsas. No es entonces suficiente deconstruir la narrativa patriótica, donde la deconstrucción se deriva en construcción y el deconstructivismo se deconstruye a sí mismo.

Un posible problema es la escasez o ausencia de perspectivas interdisciplinarias. Casi todos los autores son historiadores estrictos con escasa comprensión de los problemas y las teorías contemporáneas. En términos de estudios de nacionalismo, esto puede ser un serio inconveniente (Conversi 1995).

Aún más problemática es la ausencia de cualquier referencia a la 'gran transición' en curso, la entrada en la nueva época del Antropoceno que señala el impacto humano irreversible en la superficie geológica y los ecosistemas de la Tierra (Davies 2016; Waters et al. 2016).

Los físicos nucleares establecieron el comienzo de esta época en una fecha precisa, el 16 de julio de 1945, cuando el ejército de los Estados Unidos detonó la primera arma nuclear como parte del «Proyecto Manhattan». De hecho, un capítulo está dedicado a 1960, el año de las primeras pruebas atómicas francesas en el desierto de Argelia (Boucheron 2017: 673-677). Francia explotó 210 dispositivos, 50 de los cuales en la atmósfera. Las pruebas nucleares subsiguientes en los atolones Mururoa y Fangataufa entre 1966 y 1996 también pudieron haber desempeñado este papel, ya que el nivel de contaminación irreversible hace que las islas sigan siendo inhabitables en la actualidad: la radioactividad llegó a Perú y Nueva Zelanda en ese momento. La idea gaullista de la grandeur y del excepcionalismo francés jugó un papel fundamental aquí. No hace falta decir que la noción imprescindible de Antropoceno no se utiliza incluso en este contexto crucial. Tampoco hay ninguna referencia a la Gran Aceleración en curso (the Great Acceleration) (McNeill and Engelke 2016). 
La datación precisa es importante en el estudio de la historia. Existen desacuerdos menores entre los científicos sobre el comienzo de la nueva época, pero se están disipando: los físicos nucleares y varios geólogos se centran en los residuos nucleares, cuyo impacto se creía que era principalmente local o regional, aunque las pruebas termonucleares desencadenadas hasta 1980 dejaron una ilimitada huella. Pero muchos otros científicos se centran en el crecimiento de los patrones de consumo excesivo y el agotamiento de los recursos planetarios, particularmente el aumento en el consumo de combustibles fósiles. En este sentido, la década de 1960 se convirtió en una fecha más realista para evaluar la entrada en una nueva época como resultado del cambio climático provocado por el hombre (Moreno and Conversi 2017b; 2018). Es asombroso observar que hay más acuerdo entre los científicos duros, desde los físicos nucleares hasta los geólogos y los químicos, que entre los historiadores y politólogos, donde apenas ha comenzado el debate sobre el Antropoceno (Moreno and Conversi 2017a). También se tiene que considerar como la búsqueda de contra-tecnologías o «technofixes», pretendiendo solucionar la crisis ecológica únicamente a través del avance tecnológico (Huesemann and Huesemann 2011), promete ser una era no solo de genocidios masivos, sino de un posible «omnicidio» (Levene and Conversi 2014), o sea la potencial destrucción de todas formas vivientes.

Aún más, en un volumen dedicado a la 'historia mundial de Francia', ¿dónde está la reunión COP21? El acuerdo climático dentro de la Conferencia de las Naciones Unidas sobre Cambio Climático (UNFCCC) fue firmado por unanimidad en París por 196 países en Diciembre 2015 ¿Qué momento más cosmopolita podría haber tenido lugar en la Francia contemporánea? Esto podría haber funcionado perfectamente como capítulo final, pero sigue siendo una oportunidad perdida.

El volumen revela nuevas tendencias dentro del estudio del nacionalismo, incluso superando el constructivismo o deconstruccionismo intentando producir una nueva «historia instantánea» quizás idónea para la era de Twitter. El trabajo de Boucheron presenta todo lo contrario de los predecibles tratados repletos de Grandes Hombres y Grandes Eventos. De esta forma, hemos llegado al término con nuevas formas de re-concebir la reconfiguración de la misma identidad nacional en un sentido más internacional, con la elección de instantes o movimiento de rápidos cambios simbólicos, culturales y políticos que imparten su huella a través de las generaciones. 
Historia instantánea. El nuevo impulso historiográfico francés o historia...

\section{Referencias}

AKÇAM, Taner, The Young Turks' Crime against Humanity: The Armenian Genocide and Ethnic Cleansing in the Ottoman Empire. Princeton, N.J.: Princeton University Press, 2012.

AKÇAM, Taner, 'The Young Turks and the plans for the ethnic homogenization of Anatolia', in O. BARTOV \& E. D. WEITZ (eds.) Shatterzone of Empires. Coexistence and Violence in the German, Habsburg, Russian, and Ottoman Borderlands. Bloomington: Indiana University Press, pp. 258-280, 2013.

ArChilés CARdona, Ferran \& Marta GARCIA CARrion, «En la sombra del Estado. Esfera pública nacional y homogeneización cultural en la España de la Restauración»', Historia Contemporánea, 2012 (II), 45, pp. 483- 518.

Boucheron, Patrick (ed.), Histoire Mondiale de la France. Paris: Seuil, 2017.

BuRKe, Peter, The French Historical Revolution: The Annales School 1929-2014. Cambridge: Polity Press, 2 ed., 2015.

CONVERSI, Daniele, 'Reassessing current theories of nationalism: Nationalism as boundary maintenance and creation', Nationalism and Ethnic Politics, 1, 1, 1995, pp. 73-85.

CONVERsi, Daniele, 'Nación, estado y cultura. Para una historia política y social de la homogeneización cultural (1789-1945)', Historia Contemporánea, 45, 3, 2012, pp. 429-473.

DAVIES, Jeremy, The Birth of the Anthropocene. Oakland, CA: University of California Press, 2016.

Feyerabend, Paul, Against Method: Outline of an Anarchist Theory of Knowledge. London: Verso/New Left Books, 1975.

HoBSBAwm, Eric J. \& Terence O. RANGER (eds.), La invención de la tradición. Barcelona: Crítica, 2013

Huesemann, Michael \& Joyce Huesemann, Techno-Fix: Why Technology Won't Save Us or the Environment. Gabriola Island, BC: New Society Publishers, 2011.

LEVENE, Mark \& Daniele CONVERSI, 'Subsistence societies, globalisation, climate change and genocide: discourses of vulnerability and resilience', The International Journal of Human Rights, 18, 3, 2014, pp. 281-297.

McNeILl, John R. \& Peter Engelke, The Great Acceleration An Environmental History of the Anthropocene since 1945. Philadelphia, Pa: University of Pennsylvania Press/ Cambridge: Harvard University Press, 2016.

Moreno, Luis \& Daniele Conversi, 'Antropoceno, cambio climático y modelo social', Documentación social, 183, 2017a, pp. 13-30.

Moreno, Luis \& Daniele ConVERsi, 'Modelo social y límites al crecimiento en el antropoceno', Eunomía. Revista en Cultura de la Legalidad, 12, 0, 2017b, pp. 310-314.

Historia Contemporánea 58, 2018: 883-892 
Moreno, Luis \& Daniele Conversi, 'Cambio climático y modelo de bienestar en la era del antropoceno', in G. N. JARAíz Arroyo (ed.) Bienestar Social y Politicas Publicas. Retos para Pactar el Futuro. Madrid: Los Libros de la Catarata, pp. 74-90, 2018.

OneTO, Gilberto, L'invenzione della Padania : la rinascita della comunità più antica d'Europa. Bergamo: Foedus, 1997.

PiketTy, Thomas, Capital in the Twenty-First Century. Cambridge, Mass.: The Belknap Press of Harvard Univ. Press, 2014.

SCHALLER, Dominik J. \& Jurgen ZIMMERER (eds.), Late Ottoman Genocides: The Dissolution of the Ottoman Empire and Young Turkish Population and Extermination Policies. London: Routledge, 2009.

ÜNGÖR, Uğur Ümit, The Making of Modern Turkey. Nation and State in Eastern Anatolia, 1913-50. Oxford: Oxford University Press, 2011.

ÜngöR, Uğur Ümit \& Mehmet Polatel Confiscation and Destruction: The Young Turk Seizure of Armenian Property. London/New York: Continuum. 2011.

WATERs, C. N., et. al. , «The Anthropocene is functionally and stratigraphically distinct from the Holocene», Science, 351, 6269, 2016, pp. 2622.

WeITz, Eric D., 'Germany and the Young Turks: Revolutionaries into Statesmen', in R. G. Suny, F. M. GöçEK \& N. M. NAIMARK (eds.) A Question of Genocide: Armenians and Turks at the End of the Ottoman Empire. Oxford: Oxford University Press, pp. 175-198, 2011. 